\title{
Ketamine as a Potential Treatment for Suicidal Ideation: A Systematic Review of the Literature
}

\author{
Lael Reinstatler $\cdot$ Nagy A. Youssef
}

Published online: 10 February 2015

(c) The Author(s) 2015. This article is published with open access at Springerlink.com

\begin{abstract}
Objective To review the published literature on the efficacy of ketamine for the treatment of suicidal ideation (SI). Methods The PubMed and Cochrane databases were searched up to January 2015 for clinical trials and case reports describing therapeutic ketamine administration to patients presenting with SI/suicidality. Searches were also conducted for relevant background material regarding the pharmacological function of ketamine.

Results Nine publications (six studies and three case reports) met the search criteria for assessing SI after administration of subanesthetic ketamine. There were no studies examining the effect on suicide attempts or death by suicide. Each study demonstrated a rapid and clinically significant reduction in SI, with results similar to previously described data on ketamine and treatment-resistant depression. A total of 137 patients with SI have been reported in the literature as receiving therapeutic ketamine. Seven studies delivered a dose of $0.5 \mathrm{mg} / \mathrm{kg}$ intravenously over $40 \mathrm{~min}$, while one study administered a $0.2 \mathrm{mg} / \mathrm{kg}$ intravenous bolus and another study administered a liquid suspension. The earliest significant results were seen after $40 \mathrm{~min}$, and the longest results were observed up to 10 days postinfusion.

Conclusion Consistent with clinical research on ketamine as a rapid and effective treatment for depression, ketamine has shown early preliminary evidence of a reduction in
\end{abstract}

L. Reinstatler · N. A. Youssef

Department of Psychiatry, Medical College of Georgia at

Georgia Regents University, Augusta, GA, USA

N. A. Youssef $(\square)$

Department of Psychiatry, Charlie Norwood VA Medical Center,

1 Freedom Way, 2G100, Augusta, GA 30904, USA

e-mail: nyoussef@gru.edu depressive symptoms, as well as reducing SI, with minimal short-term side effects. Additional studies are needed to further investigate its mechanism of action, long-term outcomes, and long-term adverse effects (including abuse) and benefits. In addition, ketamine could potentially be used as a prototype for further development of rapid-acting antisuicidal medication with a practical route of administration and the most favorable risk/benefit ratio.

\section{Key Points}

Preliminary data from randomized controlled trials have demonstrated that ketamine may rapidly and effectively control treatment-resistant depression, though the effects are transient.

A small subset of studies has demonstrated similar results in the effects of ketamine on suicidal ideation.

Ketamine has potential as a rapid treatment for suicidal ideation and/or a possible model compound for future drug development.

\section{Introduction}

For decades, ketamine has been recognized as an anesthetic by the medical and veterinary worlds. It has long been used as an anesthetic for children [1] and is also occasionally used as an anesthetic for administration of electroconvulsive therapy (ECT) to patients with high seizure thresholds [2]. Its use for medical indications is attributable to its safety and wide therapeutic range [3]. 
Only recently has it been utilized for the treatment of depression and suicidal ideation (SI). First described by Berman et al. [4], ketamine has since been observed in several small randomized controlled trials to rapidly reduce depressive symptoms and SI [4-11]. Several review papers and recommendations have been written regarding application of ketamine in treatment-resistant depression (TRD), as well as for depressive episodes of bipolar disorder, but few have directly addressed its usefulness for treating SI. Nonetheless, a recent post hoc analysis of four studies found that ketamine has an impact on SI that is independent of improvement in depression and anxiety [12]. Therefore, the aim of the current review is to provide an overview of the data describing the clinical efficacy of ketamine in SI. There are no current studies that have examined ketamine for suicide attempts and suicidal behavior as outcomes.

\section{Methods}

The PubMed and Cochrane databases were searched by the two authors (L. R. and N. A. Y.) independently to select relevant studies published up to January 2015, using combinations of the terms "ketamine," "suicidal ideation," "suicide," "suicidal," and "suicidality." After all publications were obtained, the articles were individually reviewed and the following inclusion criteria were used for selection: the publication was in the English language; the patients had SI measured at baseline; and the publication was in a peer-reviewed journal. Because only a limited number of studies directly assessed SI, we included randomized controlled trials, open-label uncontrolled studies, and case series/reports. A total of 41 articles were reviewed, and nine met our inclusion criteria (Fig. 1). A combination of relevant reference citations within the original nine publications and additional searches for necessary background information completed the total data collection.

\section{Results}

A total of nine publications (six studies and three case reports) investigated the efficacy of ketamine for SI [4, 7, 10, 11, 13-17]. Three of these studies were randomized controlled trials $[4,11,18]$, three were open-label nonrandomized studies [15-17], and three were case reports $[10,13,14]$. Similar to the findings of studies examining patients with TRD, a reduction in SI was seen as early as $40 \mathrm{~min}$ and lasted for an average of 3 days. The results are summarized in Table 1 and are discussed further below.

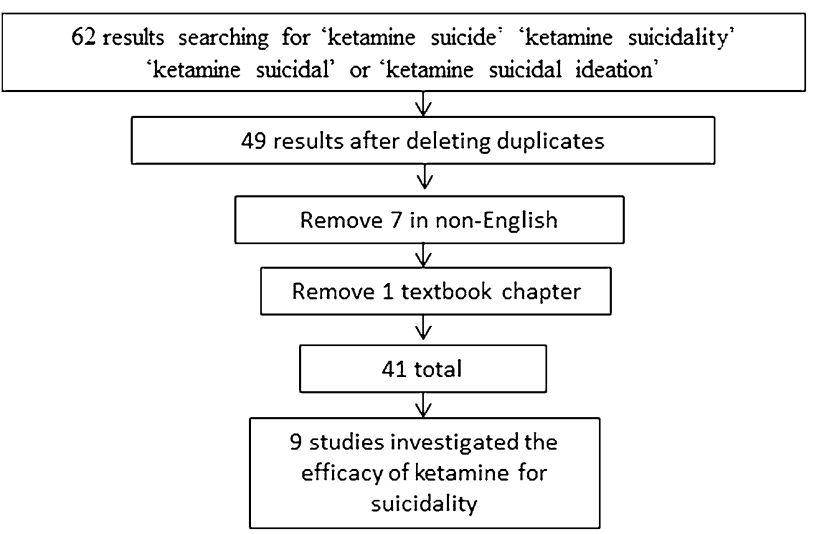

Fig. 1 Search strategy for the systematic review of ketamine as a treatment for suicidal ideation or suicidality

In the first study, by Berman et al. [4], depressive symptoms, as assessed by the Hamilton Depression Rating Scale (HDRS), were measured before and after ketamine administration. As a secondary measure, an item-level analysis of the suicidality item on the HDRS was also performed. Although the specific scores on the suicidality item were not reported, the authors reported a statistically significant reduction $(p=0.02)$ between the baseline and final scores of suicidality.

Similarly, in their randomized controlled add-on trial, Zarate et al. [7] examined SI as a secondary outcome, using the Montgomery-Åsberg Depression Scale (MADRS), HDRS, and Beck Depression Inventory (BDI) surveys. There were significant reductions in suicidality item scores in the 15 participants included in the study, beginning at $40 \mathrm{~min}$ and lasting for 3 days on the MADRS ( $p<0.001$ for all three rating scales). The effects lasted for 2 days as assessed by the HDRS, and for up to 10 days as assessed by the BDI. After the ketamine infusion, there was a statistically significant improvement in suicidality on the suicide items of all three rating scales.

An open-label study by Price et al. in 2009 [16] examined the effects of ketamine on SI, using the MADRS suicidal ideation item (MADRS-SI) and Implicit Association Test (IAT) measurements. Twenty-six patients with TRD were recruited. A subset of 13 of them had significant baseline SI (MADRS-SI score $\geq 4$ ). These patients were administered ketamine $0.5 \mathrm{mg} / \mathrm{kg}$ intravenously over $40 \mathrm{~min}$. Among the 13 patients, $8(62 \%)$ had an MADRSSI score of $\leq 124 \mathrm{~h}$ postinfusion, 3 (23\%) had a score of $2-3$, and two $(15 \%)$ remained at or above an MADRS-SI score of 4 . The overall average reduction in MADRS-SI scores was just over $2(p<0.001)$. It is interesting to report the results unique to that study related to implicit measures of suicidality. In a separate subset of 12 patients, implicit measures were assessed using the IAT, which assesses implicit suicidal associations and is a "behavioral 


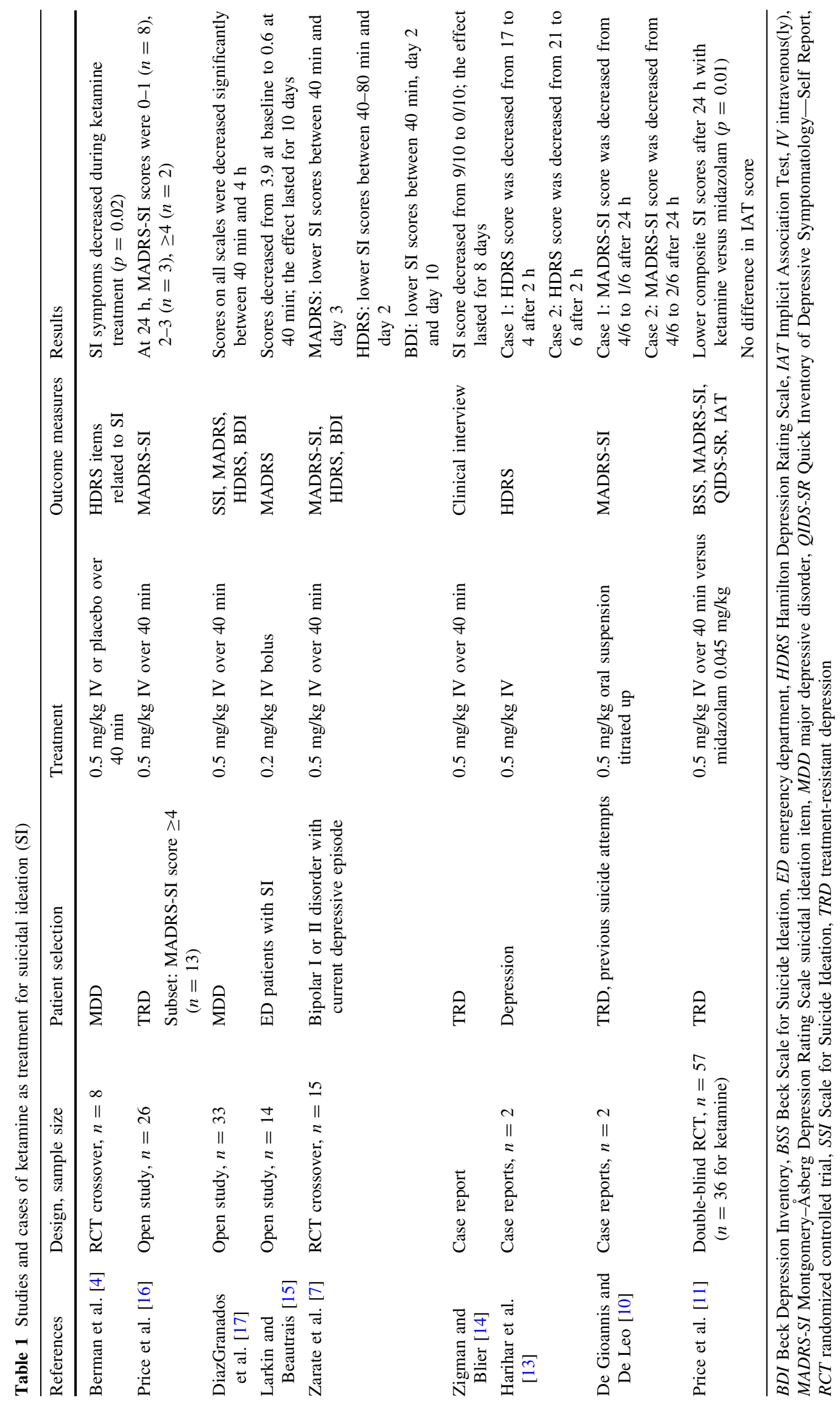


measure of suicidal cognition." Statistical analysis revealed that stronger implicit associations correlated with higher MADRS-SI scores, and the implicit measurements significantly decreased after the ketamine infusion $(p=0.003)$.

In another open-label study, 33 patients with TRD currently experiencing a major depressive episode were given ketamine $0.5 \mathrm{mg} / \mathrm{kg}$ intravenously for specific observation of its effects on SI. SI was measured on four surveys: the Scale for Suicide Ideation (SSI), MADRS, HDRS, and BDI. The authors specifically used the SSI to determine the presence of baseline suicidality, and they found that 10 of the 33 patients had significant SSI scores of $>4$. In this population, significant decreases in symptoms (SSI scores of $<4$ ) were seen beginning at $40 \mathrm{~min}$ in nine of the patients and at $80 \mathrm{~min}$ in the tenth one. The average time to an SSI score of 0 for all 10 patients was $2 \mathrm{~h}$. The improvement in their clinical symptoms lasted for up to $4 \mathrm{~h}$. When the 33 patients were analyzed together, clinical improvement was seen across all four suicide scales from 40 to $230 \mathrm{~min}(p<0.001)$ [17].

Larkin and Beautrais [15] followed 14 patients who presented with SI to the emergency department (ED). The patients received an intravenous bolus of ketamine $0.2 \mathrm{mg} / \mathrm{kg}$ over $1-2 \mathrm{~min}$. After the infusion, they were followed for 10 days and their symptoms were measured using the MADRS. These patients saw significant improvements in their clinical symptoms, beginning at $40 \mathrm{~min}$, with the MADRS-SI score decreasing from 3.9 to 0.6. The patients subjectively reported relief of SI, which lasted for the 10 days that they were followed.

In their case report, Zigman and Blier [14] described how a single infusion of ketamine $0.5 \mathrm{mg} / \mathrm{kg}$ over $40 \mathrm{~min}$ stabilized a patient with TRD and suicide intent with a plan. The patient had made a previous suicide attempt but had no other psychiatric history. She presented with an MADRS score of 48 and SI. She elected to receive ketamine rather than ECT. The patient reported immediate relief of her symptoms after the 40-min infusion. The patient reported that the effect of ketamine lasted for 8 days, and she denied any return of SI for the following month.

The second and third case reports, published by Harihar et al. [13], followed two patients with depression and suicidality. The first patient had a psychiatric history of obsessive-compulsive disorder and worsening depression, including suicidal thoughts. $\mathrm{He}$ received ketamine $0.5 \mathrm{mg} / \mathrm{kg}$ intramuscularly after admission to the hospital for a suicide attempt. His HDRS score changed from a baseline of 17 to $4,2 \mathrm{~h}$ after the ketamine treatment. The second patient had a history of recurrent major depression with recently worsening SI. After the same dose of ketamine, his HDRS score changed from 21 to 6 . Neither patient reported any side effects.
The most recent case reports documented two patients with TRD, chronic SI, and multiple past suicide attempts. Both patients were given ketamine $0.5 \mathrm{mg} / \mathrm{kg}$ in a liquid suspension. For the first patient, his dose was titrated up to $3 \mathrm{mg} / \mathrm{kg}$, where he attained a maximum response without side effects. His baseline MADRS-SI score was $4 / 6$, and $24 \mathrm{~h}$ after the ketamine treatment, it had decreased to $1 / 6$. The authors reported sustained remission with repeated oral dosing. However, no follow-up assessment was done. Similar results were seen in the second patient. The patient received a maximum dose of oral ketamine solution $1.5 \mathrm{mg} / \mathrm{kg}$. She achieved a change in her MADRS-SI score from $4 / 6$ to $2 / 6$ after $24 \mathrm{~h}$, and the authors also reported that she had sustained clinical remission of SI with monthly oral doses of ketamine [10].

The largest study to date included 57 patients with TRD in a randomized double-blind placebo-controlled trial [11]. Patients were randomized to receive either ketamine $0.5 \mathrm{mg} / \mathrm{kg}$ intravenously over $40 \mathrm{~min}$ or midazolam $0.045 \mathrm{mg} / \mathrm{kg}$ intravenously. Patients who were thought to be at imminent suicide risk were excluded from the study because of ethical considerations about using a placebo. A total of 36 patients received ketamine, and 21 received midazolam. As in the previous study conducted by this group, the patients were assessed for explicit and implicit suicidality. A combination of the Beck Scale for Suicide Ideation (BSS), MADRS-SI, and the Quick Inventory of Depressive Symptomatology-Self Report (QIDS-SR) comprised the explicit measurements in a measurement termed "SI composite scores," while the IAT looked at implicit associations. Results were compared $24 \mathrm{~h}$ after treatment. The ketamine treatment group experienced a greater reduction in SI composite scores than the placebo group ( $p=0.01)$. In addition, $53 \%$ of the treatment group scored 0 on explicit measures of suicide after $24 \mathrm{~h}$, while only $24 \%$ of the placebo group scored $0(p=0.03)$. When looking at implicit measures, the authors found no difference in the effect on implicit suicidality, based on the treatment after $24 \mathrm{~h}$. They did note that the scores for one item on the IAT, "escape = me," decreased from baseline only in the ketamine group [11].

\section{Discussion}

With an estimated prevalence of mood disorders ranging from 3.3 to $21.4 \%$ and the substantially increased risk of suicide among patients with mood disorders, treatment is certainly warranted [19]. Current treatment options for suicidality are limited. They include brain stimulation therapeutics, such as ECT, and pharmacological intervention (lithium, clozapine). The efficacy of lithium in treating suicidality has been documented $[20,21]$ and has recently 
been reviewed and pooled in a recent meta-analysis of 48 studies [22]. Clozapine has also been shown to reduce suicide risk in patients with schizophrenia [23, 24]. The limitations of both lithium and clozapine include a longer time to efficacy in this psychiatric emergency/urgency, compared with the early response to ketamine [25]. Ketamine seems to be gaining substantial evidence as a pharmacological option for depression with a fast onset of action, but its long-term effects need further investigation. In addition, ketamine probably offers a faster onset of action in terms of SI, but further work is certainly needed in this area. Given the risk of suicide and even the increasing rates of suicide in certain subgroups, such as soldiers and veterans [26, 27], there is an urgent need for faster therapeutics for SI and TRD. Importantly, suicidality and suicide pose a high global burden of patient suffering to families and society. Although several small-to-moderate sized studies, in addition to several reviews, have been published that have examined the efficacy of ketamine in TRD, there are considerably fewer published data specifically examining ketamine in patients presenting with SI. Notably, only three studies have directly examined SI as the primary outcome $[11,16,17]$, while the rest examined SI as the secondary outcome [4, 15, 18], not including case reports. This review summarizes the current published literature regarding ketamine as a treatment for SI. The data so far show promising trends of ketamine being an effective and rapid treatment with minimal side effects.

Pharmacologically, ketamine is an $N$-methyl-D-aspartate (NMDA) receptor antagonist. It has been used for anesthesia in the USA since the 1970s. At subanesthetic doses, ketamine has been shown to increase glutamate levels [3]. This mechanism is relevant, as glutamate regulation and expression are altered in patients with major depressive disorder (MDD). Studies have also demonstrated an abnormal glutamate-glutamine-gamma-aminobutyric acid cycle in patients with suicidality [28]. Furthermore, ketamine has also been shown to work on nicotinic and opioid receptors [29]. No other class of antidepressant medication works to modulate the glutamatergic system, and research continues into this, with the goal of characterizing the full mechanism of action of ketamine and perhaps developing other compounds that would have similar effects. Thus, even if the approval and marketing of ketamine as a rapidacting antisuicidal and antidepressant medication is not realized, it could well be a prototype for development of other medication(s) that retain the mechanism of action with more favorable qualities and a lesser adverse effect profile (such as a longer duration of action or less or no addictive potential). Although the mechanisms explaining the antisuicidal effect and the NMDA receptor antagonism of ketamine are still unclear, some of the initial evidence points to an anti-inflammatory action via the kynurenic acid pathway. Strong suggestions as to the causal relationship between inflammation and depression/suicidality has come from studies demonstrating that cytokines [30, $31]$ and interferon- $\beta$ [32] induce depression and suicidality. Other recent studies have added to the notion of implicating brain immune activation in the pathogenesis of suicidality. For instance, one study showed microglial activation of postmortem brain tissue in suicide victims [33]. Another study found increased levels of the cytokine interleukin-6 in cerebrospinal fluid from patients who had attempted suicide [34]. Higher levels of inflammatory markers have been shown in suicidal patients than in nonsuicidal depressed patients $[33,35]$. Inflammation leads to production of both quinolinic acid (an NMDA agonist) and kynurenic acid (a NMDA antagonist). An increased quinolinic acid to kynurenic acid ratio leads to NMDA receptor stimulation. The correlation between quinolinic acid and Suicide Intent Scale scores indicates that changes in glutamatergic neurotransmission could be specifically linked to suicidality [36].

Small randomized controlled trials have demonstrated the efficacy of ketamine in rapidly treating patients with both TRD and/or bipolar depression [4, 8, 9, 11, 16-18]. Some studies have also examined suicide items as a secondary measure in their depression rating scales [4, 7]. In total, the studies examining ketamine and TRD have nearly consistently demonstrated that ketamine provides relief from depressive and suicidal symptoms, starting at $40 \mathrm{~min}$ and lasting for as long as 5 days. Questions still remain as to the long-term effects of this treatment, how much should be administered and how often, any serious adverse effects, and the mechanism of action.

Pharmacologically, ketamine has poor bioavailability and is best administered via injection [37]. In their landmark study, Berman et al. [4] found that a subanesthetic dose $(0.5 \mathrm{mg} / \mathrm{kg})$ rapidly improved depressive symptoms. Most of the subsequent studies have delivered ketamine as a constant infusion for $40 \mathrm{~min}$ at a rate of $0.5 \mathrm{mg} / \mathrm{kg}$. Others have examined its efficacy after multiple infusions and observed similar results $[8,13,16,38]$. Currently, it is recommended that ketamine be administered in a hospital setting [39].

Ketamine is considered to be a safe compound; however, it is not without its side effects. Some studies have reported symptoms of dissociation and hallucinations, although these are usually transient [7, 14, 18]. A recent study of ketamine administration in 10 patients with obsessive-compulsive disorder reported an increase in anxiety and suicidal thoughts in two patients [40]. Notably, these patients had extensive psychiatric comorbidity, and these findings have not been duplicated. Another potential concern is the long-term effects of ketamine. Studies 
regarding outcomes associated with long-term ketamine use are sparse. The current literature refers to patients who have abused ketamine as a recreational drug. In this population, the long-term consequences have not been described, but it is clear that further studies are needed in this area [3].

The weaknesses of this review include possible bias in the way in which studies were selected for inclusionincluding publication and selection bias. The literature is limited on this topic, as we have noted in this review. However, this review includes recent data from several randomized controlled trials, including one recent doubleblind placebo-controlled trial. These trials were conducted by different groups and demonstrated similar findings. It must also be noted that differences in patient populations were not explored among the papers cited in this review. Some aspects of the efficacy of ketamine may be dependent on patient age, race, gender, etc., as evidenced by the differences in effect sizes noted among the studies, even when they used the same dosages and the same measurement tools. Larger studies with greater numbers of patients are needed to assess this further.

There are still several questions that need to be answered if standard clinical use of ketamine as an agent for treatment of SI is to be definitively pursued. Further studies are needed to better identify the best dose and delivery method of ketamine. Studies should specifically be conducted to assess SI as the primary, not secondary, outcome. Studies are also needed to further characterize the long-term benefits (if any) and the long-term risks/adverse effects, especially the abuse potential [41]. Also, there are no data on prevention of suicide attempts or death by suicide. More information is also warranted to gain a better understanding of the mechanism of action of ketamine. This information could provide guidance for further drug development, as well as further insight into the pathogenesis of suicidality. Additionally, the exploration of compounds that function similarly to ketamine warrants attention. One of these compounds may be scopolamine, an antagonist at cholinergic muscarinic receptor sites. Studies have demonstrated effective resolution of depressive symptoms within 3 days of treatment with scopolamine in some patients [42]. Also, the metabolite norketamine, which may be a possible player in the antidepressant and/or antisuicidal effect of ketamine [43, 44], deserves further research. Investigations into ketamine and other promising agents for emergent treatment of suicidality merit further research and replication in larger studies, in addition to further understanding of the mechanism of action. It should be noted that although NMDA antagonism has been proposed as the primary mechanism of action, some researchers have questioned that and raised the possibility that its stimulant action or opiate agonism might be more important than is currently appreciated [41].

\section{Conclusion}

There is early preliminary evidence not only for the antidepressant action but also for the rapid antisuicidal action of ketamine. Further research is needed to achieve better understanding of the mechanism of action and the most appropriate route of administration, to better define dosing, and to examine the long-term adverse effects (including abuse potential) and benefits of ketamine, while exploring potential similar medications that might provide better side effect profiles, or that are more easily administered, in larger double-blind randomized clinical trials.

Acknowledgments There are no conflicts of interest relevant to this report. In addition, no funding source was used in relation to this review.

Open Access This article is distributed under the terms of the Creative Commons Attribution Noncommercial License which permits any noncommercial use, distribution, and reproduction in any medium, provided the original author(s) and the source are credited.

\section{References}

1. Elliott E, et al. Ketamine anaesthesia for medical procedures in children. Arch Dis Child. 1976;51(1):56-9.

2. Hoyer $\mathrm{C}$, et al. Impact of the anesthetic agents ketamine, etomidate, thiopental, and propofol on seizure parameters and seizure quality in electroconvulsive therapy: a retrospective study. Eur Arch Psychiatry Clin Neurosci. 2014;264(3):255-61.

3. Salvadore G, Singh JB. Ketamine as a fast acting antidepressant: current knowledge and open questions. CNS Neurosci Ther. 2013;19(6):428-36.

4. Berman RM, et al. Antidepressant effects of ketamine in depressed patients. Biol Psychiatry. 2000;47(4):351-4.

5. Ibrahim L, et al. A Randomized, placebo-controlled, crossover pilot trial of the oral selective NR2B antagonist MK-0657 in patients with treatment-resistant major depressive disorder. J Clin Psychopharmacol. 2012;32(4):551-7.

6. Ibrahim L, et al. Rapid decrease in depressive symptoms with an $\mathrm{N}$-methyl-D-aspartate antagonist in ECT-resistant major depression. Prog Neuropsychopharmacol Biol Psychiatry. 2011;35(4):1155-9.

7. Zarate CA Jr, et al. Replication of ketamine's antidepressant efficacy in bipolar depression: a randomized controlled add-on trial. Biol Psychiatry. 2012;71(11):939-46.

8. Murrough JW, et al. Antidepressant efficacy of ketamine in treatment-resistant major depression: a two-site randomized controlled trial. Am J Psychiatry. 2013;170(10):1134-42.

9. Katalinic N, et al. Ketamine as a new treatment for depression: a review of its efficacy and adverse effects. Aust N Z J Psychiatry. 2013;47(8):710-27.

10. De Gioannis A, De Leo D. Oral ketamine augmentation for chronic suicidality in treatment-resistant depression. Aust N Z J Psychiatry. 2014;48(7):686.

11. Price RB, et al. Effects of ketamine on explicit and implicit suicidal cognition: a randomized controlled trial in treatmentresistant depression. Depress Anxiety. 2014;31(4):335-43.

12. Ballard ED, et al. Improvement in suicidal ideation after ketamine infusion: relationship to reductions in depression and anxiety. J Psychiatr Res. 2014;58:161-6. 
13. Harihar C, Dasari P, Srinivas JS. Intramuscular ketamine in acute depression: a report on two cases. Indian $\mathrm{J}$ Psychiatry. 2013;55(2):186-8.

14. Zigman D, Blier P. Urgent ketamine infusion rapidly eliminated suicidal ideation for a patient with major depressive disorder: a case report. J Clin Psychopharmacol. 2013;33(2):270-2.

15. Larkin GL, Beautrais AL. A preliminary naturalistic study of low-dose ketamine for depression and suicide ideation in the emergency department. Int J Neuropsychopharmacol. 2011;14(8): 1127-31.

16. Price RB, et al. Effects of intravenous ketamine on explicit and implicit measures of suicidality in treatment-resistant depression. Biol Psychiatry. 2009;66(5):522-6.

17. DiazGranados N, et al. Rapid resolution of suicidal ideation after a single infusion of an N-methyl-D-aspartate antagonist in patients with treatment-resistant major depressive disorder. J Clin Psychiatry. 2010;71(12):1605-11.

18. Zarate $\mathrm{CA} \mathrm{Jr}$, et al. A randomized trial of an $\mathrm{N}$-methyl-D-aspartate antagonist in treatment-resistant major depression. Arch Gen Psychiatry. 2006;63(8):856-64.

19. Kessler RC, et al. Lifetime prevalence and age-of-onset distributions of mental disorders in the World Health Organization's World Mental Health Survey Initiative. World Psychiatry. 2007;6(3):168-76.

20. Baldessarini RJ, et al. Decreased risk of suicides and attempts during long-term lithium treatment: a meta-analytic review. Bipolar Disord. 2006;8(5 Pt 2):625-39.

21. Kovacsics CE, Gottesman II, Gould TD. Lithium's antisuicidal efficacy: elucidation of neurobiological targets using endophenotype strategies. Annu Rev Pharmacol Toxicol. 2009;49: 175-98.

22. Cipriani A, et al. Lithium in the prevention of suicide in mood disorders: updated systematic review and meta-analysis. BMJ. 2013;346:f3646.

23. Meltzer HY, et al. Clozapine treatment for suicidality in schizophrenia: International Suicide Prevention Trial (InterSePT). Arch Gen Psychiatry. 2003;60(1):82-91.

24. Modestin J, Dal Pian D, Agarwalla P. Clozapine diminishes suicidal behavior: a retrospective evaluation of clinical records. J Clin Psychiatry. 2005;66(4):534-8.

25. Muzina DJ. What physicians can do to prevent suicide. Cleve Clin J Med. 2004;71(3):242-50.

26. Bachynski KE, et al. Mental health risk factors for suicides in the US Army, 2007-8. Inj Prev. 2012;18(6):405-12.

27. Kaplan MS, et al. Suicide among male veterans: a prospective population-based study. J Epidemiol Community Health. 2007; 61(7):619-24

28. Bernstein HG, et al. Disruption of glutamate-glutamine-GABA cycle significantly impacts on suicidal behaviour: survey of the literature and own findings on glutamine synthetase. CNS Neurol Disord Drug Targets. 2013;12(7):900-13.

29. Dda Pacheco, Romero TR, Duarte ID. Central antinociception induced by ketamine is mediated by endogenous opioids and mu- and delta-opioid receptors. Brain Res. 2014;1562:69-75.

30. Raison CL, et al. Activation of central nervous system inflammatory pathways by interferon-alpha: relationship to monoamines and depression. Biol Psychiatry. 2009;65(4):296-303.

31. Wichers MC, et al. IDO and interferon-alpha-induced depressive symptoms: a shift in hypothesis from tryptophan depletion to neurotoxicity. Mol Psychiatry. 2005;10(6):538-44.

32. Fragoso YD, et al. Severe depression, suicide attempts, and ideation during the use of interferon beta by patients with multiple sclerosis. Clin Neuropharmacol. 2010;33(6):312-6.

33. Steiner $\mathbf{J}$, et al. Immunological aspects in the neurobiology of suicide: elevated microglial density in schizophrenia and depression is associated with suicide. J Psychiatr Res. 2008; 42(2):151-7.

34. Lindqvist $D$, et al. Interleukin-6 is elevated in the cerebrospinal fluid of suicide attempters and related to symptom severity. Biol Psychiatry. 2009;66(3):287-92.

35. Janelidze $\mathrm{S}$, et al. Cytokine levels in the blood may distinguish suicide attempters from depressed patients. Brain Behav Immun. 2011;25(2):335-9.

36. Erhardt $\mathrm{S}$, et al. Connecting inflammation with glutamate agonism in suicidality. Neuropsychopharmacology. 2013;38(5): 743-52.

37. Sinner B, Graf BM. Ketamine. Handb Exp Pharmacol. 2008;182:313-33.

38. Blier P, Zigman D, Blier J. On the safety and benefits of repeated intravenous injections of ketamine for depression. Biol Psychiatry. 2012;72(4):e11-2.

39. Aan Het Rot M, et al. Ketamine for depression: where do we go from here? Biol Psychiatry. 2012;72(7):537-47.

40. Niciu MJ, et al. Two cases of delayed-onset suicidal ideation, dysphoria and anxiety after ketamine infusion in patients with obsessive-compulsive disorder and a history of major depressive disorder. J Psychopharmacol. 2013;27(7):651-4.

41. Schatzberg AF. A word to the wise about ketamine. Am J Psychiatry. 2014;171(3):262-4.

42. Drevets WC, Furey ML. Replication of scopolamine's antidepressant efficacy in major depressive disorder: a randomized, placebo-controlled clinical trial. Biol Psychiatry. 2010;67(5): $432-8$.

43. Singh NS, et al. What is hydroxynorketamine and what can it bring to neurotherapeutics? Expert Rev Neurother. 2014;14(11):1239-42.

44. van Velzen M, Dahan A. Ketamine metabolomics in the treatment of major depression. Anesthesiology. 2014;121(1):4-5. 